\title{
Biological and economic consequences heat stress due to a changing climate on UK livestock
}

E Wall, A Wreford, K Topp, D Moran

SAC, Edinburgh, United Kingdom

Email: eileen.wall@sac.ac.uk

Introduction Weather and climate can directly and indirectly determine the production and welfare of livestock. An example of a direct influence is the heat balance of livestock as average temperatures increase. Excessive heat or cold increases the metabolic energy required to maintain the animal's body temperature, thus reducing the energy available for productivity and maintaining functional fitness of the animal. This requires an understanding of how environmental stressors (e.g., temperature, humidity, thermal, air speed) can directly and adversely affect animal performance, health, and well-being when coping capabilities of the animals are exceeded and can have unfavourable economic consequences. The aim of this study was to model the change in heat stress in livestock in the UK in the future and examine the biological and economic consequences of said heat stress

Materials and methods Predictions of climate change in the UK were used to estimate the impact of heat stress on production and fitness traits in UK livestock under the medium-high climate change scenario for 2020, 2050 and 2080 (UKCIP02; Hulme et al., 2002) to $50 \mathrm{~km}^{2}$ resolution. The environmental conditions that induce heat stress were estimated by calculating using the temperature humidity index (THI) for each of 10 classes of livestock, including dairy, beef, pigs and poultry. However due to space constraints only selected results are presented in this abstract. For each class of animal the biological (production, fertility and mortality) response to heat stress was modelled using methodology presented in StPierre et al. (2003). The production (e.g., kg milk loss/animal/day, MILK), fertility (e.g., change in the number of days open/animal/year, DO) and mortality (change in monthly death rate from heat stress, MORT) losses were then scaled using UK livestock census data and present day economic costs estimated across the country and the 3 time periods.

Table 1 Annual impacts of heat stress on the duration of heat stress (hours/cow/year) and selected production, fertility and mortality in UK dairy cows by region in 2050 and 2080

\begin{tabular}{lcrrrr}
\hline \hline Region & Year & Duration & MILK & DO & MORT \\
\hline East England & 2050 & 186 & 1.3 & 0.42 & 1.25 \\
& 2080 & 601 & 21.5 & 3.19 & 2.87 \\
East Midlands & 2050 & 132 & 0.4 & 0.19 & 1.04 \\
& 2080 & 516 & 15.7 & 2.58 & 2.54 \\
North East & 2050 & 0 & 0.0 & 0.00 & 0.00 \\
& 2080 & 111 & 0.0 & 0.05 & 1.71 \\
North West & 2050 & 0 & 0.0 & 0.00 & 0.00 \\
South East & 2080 & 170 & 1.2 & 0.37 & 1.45 \\
& 2050 & 350 & 4.0 & 1.13 & 1.77 \\
South West & 2080 & 852 & 41.9 & 5.28 & 3.73 \\
Wales & 2050 & 137 & 0.6 & 0.27 & 0.87 \\
& 2080 & 495 & 15.4 & 2.33 & 2.59 \\
& 2050 & 52 & 0.2 & 0.09 & 0.37 \\
West Midlands & 2080 & 290 & 7.3 & 1.23 & 1.78 \\
Yorkshire and & 2050 & 138 & 0.3 & 0.15 & 1.23 \\
Humberside & 2080 & 2497 & 14.8 & 2.48 & 2.46 \\
\hline \hline
\end{tabular}

Results Table 1 shows that there was geographical variation in the extent to which animals suffered heat stress in the UK (e.g., dairy cows experiencing 852 hours of heat stress/year by 2080 in South East England compared to 0 hours in Northern Ireland, Scotland and Yorkshire and Humberside). These differences meant that the production, fertility and mortality losses varied both spatially and temporal in all categories of animals. Beef cows experienced less than half the duration of heat stress than dairy cows with a subsequent negligible impact on production, fertility and mortality. The average annual duration of heat stress in 2080 for monogastric animals was 92 hours for breeding sows, 214 hours of growing pigs and 420 hours of layer hens. The impact of heats stress on production was greatest is laying hens (24 g of egg loss/bird/year) followed by growing/finisher pigs (425 g reduction in liveweight gain/animal/year). The overall economic consequences of these heat stress losses were estimated to cost the UK livestock industry $£ 5.8$ million (GBP) by 2080 inproduction losses and $£ 34$ million in mortality losses. These costs exclude adaptation or extreme events (e.g., heat wave) and reflect the costs of the impacts of the gradual change of temperature (and humidity) in the UK.

Conclusions This study shows that the warming climate scenario in the UK is predicted to have a negative impact on the welfare of livestock. Not only will the animals be suffering heat stress but this will have a knock-on effect the economics of the system due to losses in production and functional performance of the animal. These represent private losses and we can expect industry to adapt if guided by appropriate regulatory reform and surveillance. The addition of extreme events to gradual climate change may provide a "shock" to livestock systems. The results show that the damages due to a warming climate will be amplified with the additional stress of extreme events, such as a heat wave. Also, some of the impacts of climate change on animal production and functionality (e.g., health and welfare) may interact with externalities and/or adaptations farmers make in other areas of the farming system (e.g., adoption of mitigation tools) needs to be monitored to ensure that both mitigation and adaptation measures are compatible and sustainable into the future. Continued development of information resources and tools will help farmers improve the resilience of their systems.

Acknowledgements The authors gratefully acknowledge funding from Defra.

References Hulme, M., Jenkins, G.J., Lu, X., Turnpenny, J.R., Mitchell, T.D., Jones, R.G., Lowe J., Murphy, J.M., Hassell, D., Boorman, P., McDonald, R. and Hill, S. 2002. Climate change scenarios for the United Kingdom: the UKCIP02 scientific report.

St-Pierre N.R., B. Cobanov, and G. Schnitkey. 2003. Journal of Dairy Science 86, (E. Suppl.),E52-E77 